\title{
Translation and analysis of Jesus Sanchez short stories
}

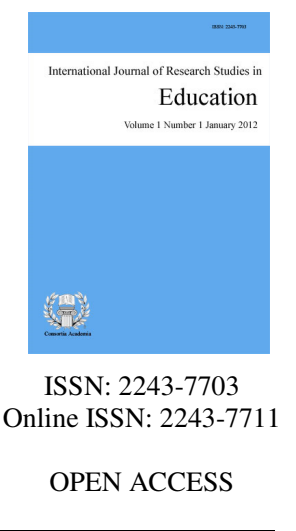

Gorumba, Jennifer $\bowtie$

Palompon Institute of Technology, Philippines (jennifer.gorumba@yahoo.com)

\section{Abstract}

Palompon, Leyte is said to have writers, as such with that of Jesus Sanchez, who deserve to be recognized in the field of literature, whose writings mirror the life and culture of the people. These may be used and may be beneficial to both students and the teachers. The study aims to translate in Filipino and analyze the short stories written by Jesus Sanchez. The result of the literary analysis and translation has become the basis of enriching the literature of the region, acknowledging these short stories and publishing it. There were four short stories which were translated to Filipino and evaluated using the theory of translation and literary analysis. The sociological and psychological analyses were used to determine the dominant culture in the short stories. The study used the descriptive-qualitative research design. The study found out that the four short stories mirror the culture of the Palomponganon people in terms of their livelihood, cooperative endeavor (bayanihan), faith, and superstitious belief. Of the three stories, the main characters were teachers. This might be accounted on the experience of the author since he was a supervisor of the Department of Education. On the other hand, the other short story reflects the struggle in life of the people and their dealings with fellow. More researchers/authors are encouraged to translate and analyze local literary works to conserve and spread it and be used by many.

Keywords: Cebuano literature; translation; literary analysis; culture; local writer; Palompon, Leyte; Philippines 


\section{Translation and analysis of Jesus Sanchez short stories}

\section{Introduksyon}

Ang panitikan ay isang mahalagang bahagi ng kultura ng isang lahi. Ito ay naglalarawan ng mga tunay na pangyayaring naganap at patuloy na nagaganap sa isang lahi o bansa. Nagbibigay ito sa atin ng mga impormasyon upang maunawaan ang mga kaugalian at ang uri ng pamumuhay ng mga tao sa iba't ibang kaalaman na nagbubunga ng kaularan pansarili man o panlipunan. Masasabi ring ang panitikan ay isang uri ng lakas na nagtulak sa atin upang tayo ay kumilos. Sa ano mang kaparaanan, ito ay nauugnay sa damdamin ng mga tao upang Makita ang katwiran at karunungan (Lorenzo, 2001).

Sinabi nga ni Arrogante, Ayuyao, at Lacanlale (2010), ang pagtuturo ng literatura ay isa sa dapat bigyan ng pagpapahalaga dahil ito ay repleksyon ng ating pagkakakilanlan, hindi mapagkakaila na ang wika at literatura ay hindi maihiwalay dahil ang wika ay ang kaluluwa at ang literatura ay ang buhay nito. Ang pagtuturo ng literatura ay may layunin ito ay ang nakapagbibigay lugod, makapagpapalaganap ng karunungan, pumupukaw ng isipan, gumigising ng damdamin at iba't ibang gawain, ang mga aral ay umaapaw at nakapagbabago ng pananaw, nagpapahalaga at higit sa lahat may layuning magbigay ng wastong kaasalan. Dagdag pa niya, bawat bansa ay may sari-sariling kultura, may sari-sariling pambansang pagkakakilanlan. Kung natural lamang sa bawat isa ang magkaroon ng sariling wika, natural din na magkaroon ang bawat isa ng sariling panitikan. Panitikang magsasalamin sa tangi at tunay na pagkatao, pagkalahi, at pagkabansa.

Sa pag-aaral ni Macan (2000), mayaman ang panitikang Filipino at ito'y hitik sa bungang karunungan ng malikhang isipan ng mga manunulat na Filipino. Mga manunulat itong nagmula sa ating mga ninuno, na gumamit ng kanilang karunungan sa pagtugon sa maraming katanungan tungkol sa kanilang karunungan sa kalikasan at sa kanilang kapaligiran. Bunga nito'y nalikha nila ang ating mayamang folkor at maikling kwento sa iba't ibang rehiyunal na wika, na sumasaklaw ng ating nabuong mito, epiko, alamat, kwentong bayan, at katutubong awit at tula. Samantalang sumusunod sa mga yapak ng ating mga malikhaing mga ninuno ang maraming manunulat na humabi ng maraming obra sa iba’t ibang genre.

Sinabi ni Batnag, Petras, at Antonio (2009) ang mga panrehiyong panitikan ay higit na nagiging kawili-wili kung ito ay naisalin sa Filipino nang sa gayo'y maintindihan ng nakararami. Napauunlad nito ang panitikang pambansa. Mahalaga ang ugnayan ng panitikang panrehiyon at panitikang pambansa. Kapwa may natatanging bisa ang dalawang ito na higit na maisasakatuparan sa pagsasalin. Ang bawat rehiyon o probinsya ng ating bansa ay may mga manunulat na nagnanais maipaabot sa mga mambabasa ang yaman ng kanilang kultura, ang damdamin at pananaw nila sa bawat naisulat na mga maikling-kwento, nobela, alamat, awitin at iba pa ngunit kung minsan ay may mga hadlang din kung paano maipararating ang mga mensahe na nakapaloob sa mga akdang panliteratura lalung-lalo na sa mga akdang rehiyunal na gamit ang bernakular na lenggwahe.

Sa pag-aaral ni Tupaz (2000), siya’y gumawa ng pagsasalin ng tulang Cebuano at ang layunin niya ay upang mapaabot sa kamalayan at kaalaman ng lahat at ng buong Pilipinas ang mga tula ng kulturang Bisayang Cebu. Dugtong pa niya na dapat maisalin sa Filipino ang mga panitikang panrehiyon at mailathala para maging bahagi ng panitikang pambansa dapat hindi lamang ang mga panitikang Tagalog o mga naisulat ng mga taga-Luzon ang dapat gamitin sa pagtuturo ng panitikan. Dapat pahalagahan din ang mga panitikang panrehiyon. Ayon nga sa konstitusyon ng 1987, sa Artikulo XIV, Sek. 9, ay nagtadhana na:

“Ang kongreso ay dapat magtatag $n g$ isang Komisyon ng Wikang Pambansa at magtalaga ng mga kinatawan ng iba't ibang rehiyon at mga didiplina na magsasagawa, mag-uugnay at magtataguyod ng mga pananalisik sa Filipino at iba pang mga wika para mapaunlad mapalaganap at mapanatili." 
Ang panitikan ay sumasalamin sa kultura ng isang lugar, tinatalakay ang iba't ibang damdamin, naglalaraawan ng buhay, pamumuhay, lipunan, pamahalaan, pananampalataya at mga karanasang kaugnay ng iba't ibang uri ng damdaming tulad ng pag-ibig, kaligayahan, kalungkutan, pag-asa, pagkamuhi, pagkapoot, paghihiganti, sindak at pangamba. Ito ay dapat pagyamanin at ituro nang wasto. Isa ring sangkap para mas lalong maging kawiliwili ang panitikan ay ang pagsusuri, ito ay masusi na paghihimay ng nilalaman ng mga akdang panitikan sa pamamagitan ng paggamit ng mga dulog ng kritisismo nang sa gayon ay mas lalong mangibabaw ang pag-unawa sa katha.

Sa pagsusuri ay binigyan ng pagpapahalaga ni Desierto (2003), nang sabihin niyang dapat pinahahalagahan natin ang isang bagay kung ito'y lagi nating tinitingnan, pinakasisiyasat at pinakaiingatan. Gayon din naman, ang pagpapahalaga sa panitikan. Anumang nasusulat na naibigan natin pagkatapos basahin ay pinakasusuri natin bilang pagpapahalaga. Masasabing kaya natin sinuri ay dahil sa nagagandahan tayo at naiibigan natin sapagkat tumama sa ating panlasa. Nais nating purihin ang may-akda sa kalakasan ng akda at bigyang-puna ang mga kahinaan nito upang lalo niyang mapaganda ang mga susunod niyang sulatin. Ang pamumuna at pagsusuri ay di pamimintas. Ito'y nagpapahalaga sa lalong ikauunlad ng panitikan na rin sa kabuuan.

Sinabi ni Villafuerte (2000), pagsusuri ng anumang akda ay dapat maging maganda ang paksa, may kalinawan ang wika at organisado ang paglalahad. Natuklasan ni Macan (2000), sa kanyang pag-aaral patungkol sa pagsasalin at pagsusuri ng mga maikling kwento ng Cebu ay na ang pag-ibig sa bayan, sa kapwa, at kagandahang-asal ang nangibabaw sa mga kaisipan ng mga maikling kwento, na may kaugnayan sa pananagutan, tungkulin, pangarap at hangarin ng isang tao. Iba't ibang damdamin ang naka-apekto sa mananaliksik tulad ng galit, awa, poot at inggit sa mga tauhan at mga pangyayarig umiikot sa buong kwento. Mga pangyayaring naglalahad ng pananagutan sa bayan, pagsuway sa mga magulang, katigasan ng ulo at bukas-kalooban na nauugnay sa kamalayang panlipunan.

Sa pag-aaral naman ni Desierto (2003), kanyang natuklasan na magiging gabay ng mga kabataan sa kanilang pakikipagsapalaran tungo sa pagbabago ang mga kwento at mapahahalagahan ang mga karanasan na siyang nagbibigay ng panibagong anyo ng kanyang buhay. Sa mga kwento ay dapat malaman ng mga mag-aaral ang mga balyu dahil may malaking implikasyon ito sa kanilang buhay at makatutulong ito sa pagtahak sa tamang landas at gayundin sa paghubog ng kanilang kaugalian tungo sa ikabubuti ng sarili at kapwa. Ayon kay Velasco (2000), sa kanyang pag-aaral, panahon na upang manumbalik at matutuhan ng ating mga kabataan sa kasalukuyan ang ating katutubong awitin, tula, sayaw, kwento, at iba pang likhang-sining na tatak ng ating pagkakakilanlan bilang isang maunlad na lipunan.

Ang mga akdang panitikan ng Palompon, Leyte partikular sa mga maikling kwento ni Jesus Sanchez ay isinalin at sinuri nang sa gayon ito maintindihan ng nakararami, lumutang ang kulturang sumasalamin sa mga maikling at magamit bilang hulwaran na kagamitang pampagtuturo, panrehiyon man o pambansa.

\subsection{Balangkas Teoritikal/Konseptwal}

Sa teorya ni Santiago (1976), sa kanyang pag-aaral sa pagsasaling wika. "Ang pagsasaling-wika ay isang sining". Madali itong maihambing o maihalintulad sa isang sining ng pagpinta. Halimbawa, ang maling laki ng guhit o ang maling pagpili at paggamit ng mga salita; ang pagkakamaling ito, tulad ng sukat, dimensyon ay pagkakamali rin sa pagbibigay ng tunay na kahulugan sa diwa ng parirala o pangungusap. Sa mga aralin ngayon ay unti-unti nang kinikilala ang mga akdang Rehiyunal, ibig sabihin ay hindi na lang nakatutok sa mga akda ng mga Katagalugan o sa bandang Luzon. Ayon pa nga kay Santiago (1976), na lalong magiging maganda kung makabubuo tayo ng tinatawag na pambansang panitkan o literatura ng bayan na kumakatawan sa kabuuan ng pambansang kaisipan. Isang panitikang matatawag na kaisipang Pilipino na hango sa alinmang bilang Pilipino. Ipinapakita rito ang isang ilustrasyon buhat sa teorya ng pagsasalin ni Santiago. 


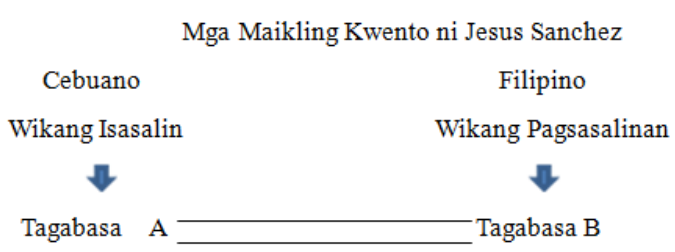

Pigura 1. Ilustrasyon buhat sa teorya ng pagsasalin ni Santiago

Ang pagsasalin ay isa sa pinakamabisang pamamaraan para maihatid ang mensahe. Sa panitikan ay maraming pamamaraan para maihatid ang mensahe ng nilalaman ngunit magkakaroon ng kahirapan kung ang wika mismo ang nagiging hadlang para maisalin ang mensahe ng mga akda. Nakikita at napapansin ng mananaliksik sa kanyang klase na kulang ang kaalaman nila kung pagbabasehan ang mga akdang naisatitik sa bayan ng Palompon. Kung ang mga akdang ito ay maisasalin sa wikang Filipino ay higit na kapaki-pakinabang.

Sa teoryang pagsusuri naman ni Berelson (1971) ay tumutukoy na ang pagsusuri ng nilalaman ay karaniwang ginagawa sa kakaunti at di-kompletong mga halimbawa o kalahok na ang mga datos na susuriin ay iilan lamang upang mabigyan ng katuturan ang tema at wastong pag-aaral. Dugtong pa niya sa pagsusuri at pag-iinterpreta ng nilalaman na nahihinuha ang epekto at kalalabasan nito upang maging bahagi ng prosesong pagsusuri.

Ito ay sinang-ayunan din ng teorya ni Alejandro, na ang pagsusuri ay dapat taglayin ng isang kritiko ang ganap na kaalaman sa kanyang paksa. Una, Ito'y tumutukoy sa kabuuan at nilalaman ng akda at ang paksang tinutukoy ng awtor. Dapat din malaman na ang buhay at kaisipan ng awtor ay isaalang-alang ang panahon ng pagkakasulat nito; ikalawa, ang katapatan ng loob ay isang katangiang dapat taglayin ng isang kritiko; ikatlo, ang isang kritiko ay dapat mag-angkin ng likas na pagkukuro. Ang isang tagasuring may likas na pagkukuro sa buhay ng awtor ay hindi sanligan sa pagkilatis ng isang akdang pampanitikan.

Sa pagsusuri ng mga maikling kwento ni Jesus Sanchez ay ginamit ang teoryang sosyolohikal at sikolohikal. Ang pinag-aaralan sa teoryang sosyolohikal ay ang mga dapat sundin sa lipunan at ang mga proseso na nagbibigkis at naghihiwalay sa mga tao hindi lamang bilang mga indibidwal kung hindi bilang kaanib ng mga institusyon, grupo at asosasyon. Ito rin ay tumutukoy sa pag-aaral ng mga buhay na lipunan ng mga tao, at kumunidad na kung saan ay naginging paksa sa sosyolohikal ang mga pag-uugali ng indibidwal hanggang sa pakikitungo sa pandaigdigang lipunan.

Samantala, ang teoryang sikolohikal ay tumutukoy sa mga akdang pampanitikan na nagpapahayag sa iba't ibang damdamin at katauhan ng may-akda. Sinusuri rin dito ang mga ugali ng mga tauhan, kaisipan at kilos, maging ang kaugnayan ng bawat isa. Dito rin mlalaman ang mga pagpababago ng dating paniniwala kung bakit tinalikuran ang dating mga karanasan gaya ng mga adhikain at mga pinaglalaban at mga pangyayaring nakaapekto sa paniniwala. Layunin ng teoryang sikolohikal ang maipaliwanag kung paano naipapakita ng isang tauhan sa akdang pampanitikan ang kanyang mga paniniwala, pananaw, pag-uugali at pagkatao.

\subsection{Layunin ng pag-aaral}

Ang layunin ng pag-aaral na ito ay ang pagsasalin at pagsusuri ng mga maikling kwento na isinulat ni Jesus Sanchez ng Palompon, Leyte. Ito ay magiging batayan sa pagpapayaman ng Wikang Filipino at makilala ang mga akdang panitikang lokal para sa pandalubhasaang pagtuturo at pagkatuto sa asignaturang Filipino.

1. pagsasalin sa Filipino ng mga maikling kwento ni Jesus Sanchez;

2. pagsusuri sa mga maikling kwento gamit teoryang sosyolohikal at sikolohikal;

3. pagtukoy at pagsusuri sa mga kulturang nangibabaw sa kwento; 
Translation and analysis of Jesus Sanchez short stories

4. paggamit sa mga naisalin at nasuring kwento sa asignaturang Panitikang Rehiyon, Pagsusuring panitikan at iba pang araling pampanitikan at mailathala ang mga naisaling maikling kwento.

\section{Metodolohiya}

Kwalitatib-Diskriptib ang ginamit sa pag-aaral para mailarawan at mailahad nang mabuti ang mga ispisipikong mga pangyayari sa kwentong sinuri. Apat na mga maikling kwento na isinulat ni Jesus Sanchez ng Palompon, Leyte ang ginamit sa pag-aaral na ito. Ginamit ang teoryang Sosyohikal at sikolohikal sa pagsusuri na naging hulwaran sa pagsisiyasat sa mga nagibabaw na mga kulturan sa kwento nang sa gayun matukoy ang pakikitungo sa panlipunan ng mga tao at grupo.

Ang Palompon, Leyte ay may maraming panitikang naisulat ngunit pinili lamang ng mananaliksik ang mga maikling kwentong nailathala. Isa na rito ang mga maikling kwento ni Jesus Sanchez. Bago pa man nakapagsalin ang mananaliksik ay pumunta sa may-akda para humingi ng pahintulot at humingi ng kopya ng kanyang mga akdang panitikan. Iminungkahi ng ng may-akda ang pagsasalin ng kanyang apat na maikling kwento. Pagkatapos maisalin ang mga kwentong: "Sa Gabiing Matidlom ang mga Gutlo sa Karaang Panahon" (Sa Gabing Madilim ang mga Oras ng Lumang Panahon) [17], "Gahong sa Baboyng Ihalas” (Bitag sa Baboy Ramo) [18], "Sa Gabiing Nagtukaw ang mga Anino" (Sa Gabing Gising ang mga Anino) [19], "Sa Dapit nga Walay Ingon” (Sa Lugar na Walang Katulad) [20] ay ipinawasto at ipanabalida sa apat na propesor na eksperto sa wika ng Palompon Institute of Tecnology.

\section{Mga resulta at diskusyon}

Mula sa masusing pagsusuri sa mga maikling kwento, maraming mga isyung sosyolohikal at sikolohikal ang nangibabaw na naging lunsaran sa pagtukoy sa mga kultura ng mga Palomponganons. Sa sosyolohikal, natuklasan sa mga maikling kwentong isinalin na ang mga gurong itinalaga sa malalayo o sa bulubunduking lugar ay ipinakikila sa Kapitan ng baranggay at pansamantalang pinapatira hanggang sa maging kabisado na niya ang lugar at mamamayan. Tatlo sa mga maikling kwento ang tumalakay nito. Ang mga pangyayari sa kasalukuyan ay talagang sumasalamin sa mga kwento dahil mapaghanggang ngayon ay ginagawa pa ito ng mga taga-baryo. Ang pagkalinga ay hindi masusukat, mula sa mga opisyal ng baranggay, maging ang mga magulang ng mga estudyante. Ang pagtanggap naman sa mga bisitang tagasuri sa mga paaralan ay talaga namang nakakabilib dahil mula sa pagdating hanggang sa pagkatapos ng mga gawain ay nakaalalay ang mga guro gayon din ang mga taga-baranggay at ang pagkaing hinanda ay abunda.

Tinalakay rin ang pagpapakitang gilas sa mga kaibigan o sa mga babae para mapalapit pero ang hindi rin maiiwasan na may mga umiiwas kahit gumastos pa ng malaki lalong-lalo na kung hindi gusto ang tao. Nagkaroon lang ng pagkakasundo kung pareha ang hilig katulad ng lamang ang pagkahilig ng manok na panabong. Isa sa mga maikling kwento ay tumalakay patungkol sa pakikihalubilo sa mga taga-baranggay sa pagtungga ng tuba bilang pampalipas oras ng guro. Ang espirito ng tuba ay nagdala sa tauhan sa di magandang gawain gaya ng pakikiapid.

\subsection{Sikolohikal}

Reincarnation, maaaring may mga taong nakaranas nito na parang bang ang mga pagyayari ay naramdaman na, gaya ng isang kwento nang siya ay matalaga sa isang baryo ng Palompon ay may naramdamdaman siya sa lugar at ang nakilalang dalaga ay parang may kaugnayan sa kanya.

Ang salitang "sigurado na", ito na marahil ang gasgas na salita na ating maririnig kapag kulang ng tiwala sa kausap. Kahit pa man kadugo, kapag pera o may ibinabalita, palaging sinasambit ang salitang ito, isa sa mga kwento ang tumalakay sa paninigurado ng babae kung maibibigay ba ang dote na hinihingi ng kanyang amain.

Sa kwentong "Bitag sa Baboy Ramo" ay nagpakita ng mga matinding damdamin, ang pagkatakot na baka 
Gorumba, J.

hindi matuloy ang kasal. Takot sa kanyang amain sa mga mapagnasang tingin. Pangamba rin ng kasintahang lalaki na baka hindi niya maibigay ang doteng hinihingi sa kanya dahil unti-unting inuubos ng mga baboy ramo ang kanyang mga pananim na mais.

"Panakaw-nakaw na kasiyahan". Damdaming hindi napigilan ng dalawang aninong pinag-isa sa dilim habang ang asawa ay nasa dagat para maggantaaw isang uri ng pangingisda sa madilim na gabi. Dahil sa mga pangyayaring ito, hindi maiiwasan ang paghihigante na hahantong sa pagpatay.

Ang tunay na pagmamahal ay maaaring magpabago sa tao sa kanyang mga desisyon. Isa sa mga kwento ang tumalakay sa isang wagas na pagmamahal ng mga ka-nayon at gayun din ang sa babaeng pumana sa puso. Ang guro sa kwento na binigyan na ng pagkakataon para mailipat na malapit sa bayan pero ito ay tinanggihan dahil nadama na niya na nasa paraiso siya dahil sa di-masukat na pagmamahal sa kanya ng mga tao.

\subsection{Kultura}

Ang mga maikling kwentong sinuri ay sumasalin sa kultura ng Palomponganons, ito marahil ay nasaksihan ng manunulat o di-kaya'y kanyang naranasan gaya na lang ng buhay ng mga guro, kabuhayan ng mamamayan, pag-uugali, paniniwala, mga gawain at iba pa.

Hospitable at Mapagbigay. Naging tradisyon na, ang pagbisita sa mga paaralan para sa isang pagsusuri. Dito pinaranas sa mga Superbisor ang taos-pusong pagtanggap sa kanila. Ang kaugaliang ito ay hindi lamang ipnamamalas para sa mga may katungkulan, pati na rin sa mga ordinaryong tao. Halos lahat din ng mga pari at nanunungkulan sa gobyerno na itinalaga sa Palompon ay sobra ang kaligayahan at pasasalamat sa ipinapakitang kabutihan at ang pagiging mapagbigay. Ang Palompon, Leyte ay isang bayan na masasabing ang masarap balik-balikan dahil sa ganitong pag-uugali.

Pagsasabong ng Manok. Malalim na nakatanim sa kulturang Pilipino ang pagsasabong. Ito ay sinasabing pampalipas-oras. Popular ang sabong sa Pilipinas pero mas maraming ang tumatangkilik nito sa mga probinsya, isa na rito ang Palompon na kahit sa pinakamaliit na barangay ay may sabong lalo-lalo na kung papalapit na ang pista.

Dote. Isa sa kwento ang tumalakay sa paghingi ng dote bago ikasal ang babae. Maaaring hindi na ito ngayon ginagawa nang karamihang mga Pilipino pero may mangilan-ngilan pa rin ang gumagawa nito sa kasalukiyan sa bayan ng Palompon.

Delicadeza. Isang ugali na kung minsan mapapatanong tayo kung marami pa ba ang mayroon nito. Sa kwentong "Bitag sa Baboy Ramo" ay magpahalik o papasukin ang lalaki sa loob ng kanilang dampa dahil natatakot sa sasabihin ng iba. Ang pag-uugaling ito ay mararamdaman o makikita pa ngayon, ang pagpapahalaga sa sarili ng mga kababaihan.

Utang na loob. "Hindi makaalis-alis ang pangunahing tauhan sa kanyang kinikilalang magulang dahil utang niya ang kanyang buhay". Halos lahat ng tao ay nakaranas na nito. Kung minsan ito ang nagiging hadlang sa pag-unlad.

Ritwal. Isa sa mga gawain ng mag-asawa sa kwento ang maghubad habang itinatanim ang mga binhi na nakaharap sa buwan nang sa gayon ang magiging ani nila ay maging sinlaki ng buwan. Marami pa rin sa atin ang naniniwala sa mga ritwal tuwing magtatanim o sa kahit anumang gawain. Sa Palompon ay mayroon pa ring gumagawa nito pero hindi nakahubad kundi may mga kinakatay na hayop bilang alay o kaya'y nag-oorasyon.

Bayanihan. Ang pagiging matulungin ay likas na kaugalian ng mga Palomponganons, kahit sa di gaanong kilala kapag nangangailangan ng tulong ay bukas palad na naglilingkod.

Tuba. Isa sa mga ikinabubuhay ng ilan sa mga residente ng Palomopon. Hindi lang ganun ka sikat gaya sa ibang mga lugar ng Leyte pero nakapagpapatawid ng buhay. 
Kamoteng Baging. Isa ring kabuhayan sa parteng bulubundukin ng Palompon. Ang mga ito ay ibenibenta tuwing Lunes na kung tawagin ng mga tao na "Tabo" na ibig sabihin ay "Market day"

\section{Konklusyon at rekomendasyon}

Ang pag-aaral na ito napakahalaga sa mga guro sa panitikan lalong-lalo na sa mga nagtuturo sa elementary at sekondarya na sumasaklaw na sa contextualization. Sa pamamagitan ng mga isinaling mga maikling kwento ay mas mabibigyang puwang ang mga lokal na manunulat at maiugnay ang mga nilalaman ng mga kwento sa aktwal na nakikita at nararanasan ng mga estudyante gaya ng kultura ng lugar. Kapakipakinabang din ito sa mga nagpapakadalubhasa sa Filipino dahil ito ay magiging gabay nila sa pagsasalin at pagsusuri nang sa gayon ay mapalaganap din nila ang mga akdang panitikang lokal. Iminumungkahi na marami pang magsasalin at magsusuri ng mga lokal na akdang panitikan nang sa gayon ito ay mapalaganap at magamit, lalong-lalo na sa mga gurong nagtuturo ng Panitakang Rehiyon at Panunuring Pampanitikan. Ang mga naisaling mga kwento ay maaari ring gawan ng modyul.

\section{Sanggunian}

Arrogante, J., Ayuyao, N. G., at Lacanlale, V. M. (2010). Panitikang Filipino: Antolohiya. Mandaluyong City: National Book Store.

Batnag, A., Petras, J., at Antonio, L. (2009). Teksbuk sa pagsasalin. Quezon City: C\&E Publishing, Inc.

Berleson, B. (1971). Analysis in communication research. New York: Hafner Publishing Company.

Desierto, E. (2003). Isang pagsusuring pampanitikan ng mga piling kwentong nagwagi sa Gawad Carlos Palanca 1950-1989 (Unpublished doctoral dissertation). Leyte Normal University, Cebu City.

Lorenzo, C. (2001). Literatura ng iba't ibang rehiyon ng Pilipinas. Makati City: Grandwater Publications and Research Corporation.

Macan, R. S. (2000). Isang pagsusuring kritikal sa limang piling maikling kwentong Cebuano (Unpublished doctoral dissertation). Cebu Normal University, Cebu City.

Sanchez, J. (1885). Sa gabing matidlom ang mga gutlo sa karaang panahon (pp. 1, 18, 19). Cebu City: Bisaya Magazine.

Sanchez, J. (1969). Gahong sa baboyng ihalas (pp. 3-10). Cebu City: SUGO Magazine.

Sanchez, J. (1982). Sa dapit nga walay ingon (pp. 31-36). Cebu City: Bisaya Magazine.

Sanchez, J. (1984). Sa gabi nga nagtukaw ang mga anino (pp. 3-5). Cebu City: Bisaya Magazine.

Santiago, A. O. (1976) Sining ng pagsasaling wika. Manila: Rex Bookstore Inc.

Tupaz, R. (2000). Gawain ng pag-aaral batay sa pagsasalin at pagsusuri ng mga piling literatura ng Rehiyon VIII (Unpublished dissertation). Leyte Institute of Technology, Tacloban City

Velasco, L. (2000). Pagpapakahulugang kultural ng mga waray sa panulaan ni Agustin EIO Mora: Mungkahing gawaing pagtuturo sa edukasyong pagpapahalaga (Unpulished masteral thesis). Leyte Normal University, Tacloban City.

Villafuerte, P. V. (2000). Panunuring pampanitikan. Valenzuela City: MEGA-JESTA Prints, Inc. 
Gorumba, J. 